K. Ichihara

Nagoya Math. J.

Vol. 166 (2002), 93-115

\title{
BIRTH AND DEATH PROCESSES IN RANDOMLY FLUCTUATING ENVIRONMENTS
}

\author{
KANJI ICHIHARA
}

\begin{abstract}
A birth and death process in a time-dependent random environment is introduced. We will discuss the recurrence and transience properties for the process.
\end{abstract}

\section{$\S 1$. Introduction and results}

Let $E$ be a finite subset of a hyperplane in $\mathbb{R}^{2 d}$ whose element has positive components. Each element in $E$ is denoted by $a=\left(\lambda_{+}^{1}(a), \lambda_{-}^{1}(a), \ldots\right.$, $\left.\lambda_{+}^{d}(a), \lambda_{-}^{d}(a)\right) \in E$.

Suppose we are given a stationary, continuous time, irreducible Markov chain $a_{t}$ on $E$. On a probability space $(\Omega, \mathfrak{F}, P)$, construct a family of independent copies of $a_{t}$, indexed by $x \in \mathbb{Z}^{d}$, the $d$-dimensional space lattice. Call these processes $a_{t}(x)$. Set $\mathbf{a}_{t}=\left(a_{t}(x)\right)_{x \in \mathbb{Z}^{d}}$.

For a given realization $\left\{\mathbf{a}_{t}, t \geq 0\right\}$, define a jump process $X_{t}$ as the nonstationary Markov process with state space $\mathbb{Z}^{d}$ and transition probability determined by

$$
\left\{\begin{array}{l}
P\left\{X_{t+h}=x \pm e_{i} \mid X_{t}=x\right\}=h \lambda_{ \pm}^{i}\left(a_{t}(x)\right)+o(h) \\
P\left\{X_{t+h}=x \mid X_{t}=x\right\}=1-h \sum_{i=1}^{d}\left\{\lambda_{+}^{i}\left(a_{t}(x)\right)+\lambda_{-}^{i}\left(a_{t}(x)\right)\right\}+o(h)
\end{array}\right.
$$

where $e_{i}=(0, \ldots, \stackrel{i}{1}, \ldots, 0) \in \mathbb{Z}^{d}$. This process $X_{t}$ is called a birth and death process in the randomly fluctuating environment $\left\{\mathbf{a}_{t}, t \geq 0\right\}$. In this paper we shall discuss the recurrence properties of the process $X_{t}$. The paired process $\left(X_{t}, \mathbf{a}_{t}\right)$ will be used to investigate the problem.

It is to be mentioned that another type of processes in randomly fluctuating environments have been introduced by Madras [4]. In the paper the recurrence and transience problem in one dimension was treated.

Received February 8, 2001.

2000 Mathematics Subject Classification: 60K37. 
Before proceeding to the existence of the above two processes, we shall introduce some notations and definitions.

Let $p_{t}(a, b)$ be the transition probability of the Markov process $a_{t}$. Since the process $a_{t}$ is irreducible on the finite state space, it has the unique invariant probability measure $\mu$. It is well known that there exists a positive constant $\alpha$ such that

$$
\left|p_{t}(a, b)-\mu(b)\right| \leq e^{-\alpha t}
$$

for $t>0$ and $a, b \in E$. Define $\boldsymbol{\mu}=\mu \stackrel{\mathbb{Z}^{d}}{\otimes}$ as the direct product of $\mu$ on $\mathbb{Z}^{d}$. Let $E^{\mathbb{Z}^{d}}$ be the space of configurations of jump rates on $\mathbb{Z}^{d}$ and let it be endowed with the usual direct topology. Then $\mathbf{a}_{t}$ is regarded as a Markovian system as in e.g. Liggett [3] and $\boldsymbol{\mu}$ is its unique invariant probability measure. Let $G$ be the infinitesimal generator of $\mathbf{a}_{t} . P_{\mathbf{a}}$ denotes the probability law of $\mathbf{a}_{t}$ starting at $\mathbf{a}_{0}=\mathbf{a}$. Set

$$
P_{\boldsymbol{\mu}}\left(B_{1} \times B_{2}\right)=\int_{B_{1}} P_{\mathbf{a}}\left(B_{2}\right) \boldsymbol{\mu}(d \mathbf{a}) .
$$

Define a difference operator $L$ on $\mathbb{Z}^{d} \times E^{\mathbb{Z}^{d}}$ by

$$
\begin{aligned}
L f(x, \mathbf{a})=\sum_{i=1}^{d}\left\{\lambda_{+}^{i}\right. & (a(x))\left(f\left(x+e_{i}, \mathbf{a}\right)-f(x, \mathbf{a})\right) \\
& \left.+\lambda_{-}^{i}(a(x))\left(f\left(x-e_{i}, \mathbf{a}\right)-f(x, \mathbf{a})\right)\right\} .
\end{aligned}
$$

We now explain briefly about the existence of the above processes $X_{t}$ and $\left(X_{t}, \mathbf{a}_{t}\right)$. Let $\eta_{t}(x),(t, x) \in[0,+\infty) \times \mathbb{Z}^{d}$ be a function with values in $E$ which is right continuous and has left limits in $t$. Set $\boldsymbol{\eta}_{t}=\left\{\eta_{t}(x)\right\}_{x \in \mathbb{Z}^{d}}$ and $\theta$. denotes the shift of $\eta$ i.e. $\theta_{s} \eta_{t}(x)=\eta_{t+s}(x)$. Define

$$
\begin{aligned}
L_{t}^{\boldsymbol{\eta}} \cdot f(x)=\sum_{i=1}^{d}\left\{\lambda_{+}^{i}\left(\eta_{t}(x)\right)\left(f\left(x+e_{i}\right)-f(x)\right)\right. \\
\left.+\lambda_{-}^{i}\left(\eta_{t}(x)\right)\left(f\left(x-e_{i}\right)-f(x)\right)\right\}
\end{aligned}
$$

Then as in Ethier and Kurtz [1], page 163-164, we can easily construct a (nonstationary) Markov process on $\mathbb{Z}^{d}$ associated with the operator $L_{t}^{\boldsymbol{\eta}} \cdot$. Denote the process by $X_{t}^{\boldsymbol{\eta}}$. and its transition probability by $p^{\boldsymbol{\eta}} \cdot(s, x ; t, y)$. Then $X_{t}^{\mathbf{a}}$ is nothing but the process introduced in (1). 
Define a family of operators $\left\{T_{t}, t \geq 0\right\}$ on $\mathbb{C}_{0}=\mathbb{C}_{0}\left(\mathbb{Z}^{d} \times E^{\mathbb{Z}^{d}}\right)$, the space of all continuous functions on $\mathbb{Z}^{d} \times E^{\mathbb{Z}^{d}}$ vanishing at the infinity, by

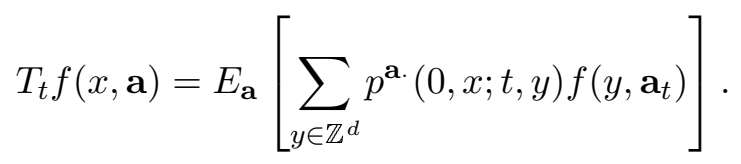

Then $\left\{T_{t}\right\}_{t \geq 0}$ is a Feller semigroup on $\mathbb{C}_{0}\left(\mathbb{Z}^{d} \times E^{\mathbb{Z}^{d}}\right)$. Indeed, we have

$$
\begin{aligned}
& T_{t+s} f(x, \mathbf{a})
\end{aligned}
$$

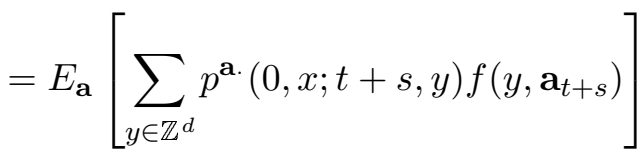

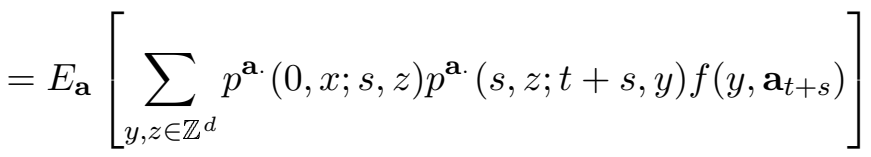

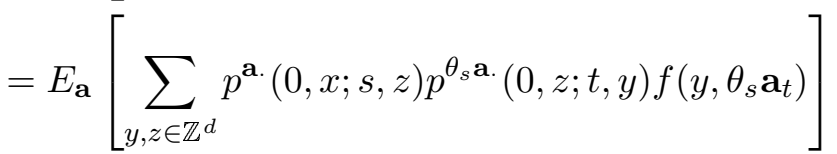

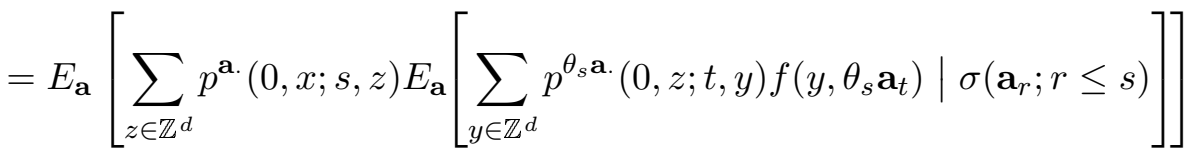

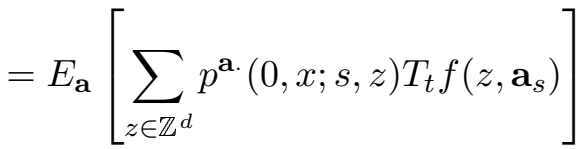

$$
\begin{aligned}
& =T_{s} \cdot T_{t} f(x, \mathbf{a}) \text {. }
\end{aligned}
$$

It is also easy to see that $T_{t} f \in \mathbb{C}_{0}$ if $f \in \mathbb{C}_{0}$. Furthermore it can be shown that the infinitesimal generator of $\left\{T_{t}\right\}$ is $\Delta=L+G$. This semigroup is associated with the bichain $\left(X_{t}^{\mathbf{a} .}, \mathbf{a}_{t}\right)$.

Set $\lambda_{ \pm}^{i, 0}=\int_{E} \lambda_{ \pm}^{i}(a) \mu(d a)$. Let $X_{t}^{i}$ be the $i$-th component of $X_{t}$. Denote by $P_{x}^{\mathbf{a}}(\cdot)$ the probability law of the birth and death process $X_{t}^{\mathbf{a}}$ starting at $x$, in the randomly fluctuating environment $\left\{\mathbf{a}_{t}, t \geq 0\right\}$.

We are now in a position to state our first result.

TheOREM 1. Suppose $\lambda_{+}^{i_{0}, 0}>\lambda_{-}^{i_{0}, 0}$ for some $i_{0} \in\{1, \ldots, d\}$. Then there exists a positive constant $\alpha_{0}^{d}$ depending only on $\lambda_{ \pm}^{i}, i=1, \ldots, d, d$ 
and $E$ such that if $\alpha>\alpha_{0}^{d}$,

$$
P_{0}^{\mathbf{a} .}\left[\lim _{t \rightarrow+\infty} X_{t}^{i_{0}}=+\infty\right]=1 \quad \text { a.s. } \quad P_{\boldsymbol{\mu}}
$$

The environment treated in Theorem 1 is biased in some sense to one direction. Thus we shall next consider the case where the environment is statistically balanced i.e. $\lambda_{+}^{i, 0}=\lambda_{-}^{i, 0}$. This case is more delicate. Therefore we need to put stronger assumptions on the environment;

There exist positive constants $p_{i}, q_{i}\left(p_{i} \neq q_{i}\right), i=1, \ldots, d$ such that

$$
E=\left\{\left(\lambda_{+}^{1}, \lambda_{-}^{1}, \ldots, \lambda_{+}^{d}, \lambda_{-}^{d}\right) ;\left(\lambda_{+}^{i}, \lambda_{-}^{i}\right)=\left(p_{i}, q_{i}\right) \text { or }\left(q_{i}, p_{i}\right)\right\}
$$

and the transition probability of the Markov chain $a_{t}$ on $E$ is given by

$$
p_{t}\left(a_{1}, a_{2}\right)= \begin{cases}\frac{1}{2^{d}}\left(1-e^{-\alpha t}\right), & a_{1} \neq a_{2} \\ \frac{1}{2^{d}}\left(1+\left(2^{d}-1\right) e^{-\alpha t}\right), & a_{1}=a_{2} .\end{cases}
$$

In this case, $\mu(a)=\frac{1}{2^{d}}$ for $a \in E$. Under the assumptions (I) and (II),

$$
\begin{gathered}
\lambda_{+}^{i, 0}=\lambda_{-}^{i, 0}=\frac{p_{i}+q_{i}}{2}, \quad i=1, \ldots, d . \\
\left(\equiv \lambda^{i, 0}\right)
\end{gathered}
$$

We have

Theorem 2. Let $d=1$. Suppose the conditions (I) and (II) hold. Then there exists a positive constant $\beta_{0}^{1}$ depending only on $p_{1}, q_{1}$ such that if $\alpha>\beta_{0}^{1}$,

$$
P_{0}^{\mathbf{a} .}\left[X_{t}=x \quad \text { for some } t>0\right]=1 \quad \text { a.s. } \quad P_{\boldsymbol{\mu}}
$$

for any $x \in \mathbb{Z}^{1}$.

Theorem 3. Let $d \geq 3$. Suppose the conditions (I) and (II) hold. Then there exists a positive constant $\beta_{0}^{d}$ depending only on $p_{i}$ and $q_{i}, i=$ $1, \ldots, d$ such that if $\alpha>\beta_{0}^{d}$,

$$
P_{0}^{\mathbf{a} .}\left[\lim _{t \rightarrow+\infty}\left|X_{t}\right|=+\infty\right]=1 \quad \text { a.s. } \quad P_{\boldsymbol{\mu}}
$$


In order to handle the two dimensional case, a further restriction on the environment is required.

$$
p_{1}=p_{2}(\equiv p) \quad \text { and } \quad q_{1}=q_{2}(\equiv q) .
$$

TheOREM 4. Let $d=2$. Suppose the conditions (I), $\left((\mathrm{I})^{\prime}\right)$ and (II) hold. Then there exists a positive constant $\beta_{0}^{2}$ depending only on $p$ and $q$ such that if $\alpha>\beta_{0}^{2}$,

$$
P_{0}^{\mathbf{a} .}\left[X_{t}=x \quad \text { for some } t>0\right]=1 \quad \text { a.s. } \quad P_{\boldsymbol{\mu}}
$$

for any $x \in \mathbb{Z}^{2}$.

It is to be remarked that all the constants $\alpha_{0}^{d}, \beta_{0}^{d}$ can be explicitly identified.

Note that it suffices to prove all the theorems under the law of the bichain $\left(X_{t}^{\mathbf{a} .}, \mathbf{a}_{t}\right)$ instead of the law of the original process.

The proofs of our theorems are based on Lyapunov's method. We shall construct Lyapunov functions by a perturbation of the ones for simple random walks. The point is to apply a sort of renormalization technique through the generator $G$.

\section{§. Proof of theorems}

Let $P_{(x, \mathbf{a})}$ be the probability law of $\left(X_{t}, \mathbf{a}_{t}\right)$ starting at $(x, \mathbf{a})$.

We start with;

[1] Proof of Theorem 1. Assume $i_{0}=1$ and set $x=\left(x_{1}, \ldots, x_{d}\right)$. In order to prove Theorem 1, we need the following two lemmas.

Lemma 1. We have

$$
P_{(x, \mathbf{a})}\left[X_{t}^{1}=0 \text { for some } t>0\right]=1
$$

for any $x=\left(x_{1}, \ldots, x_{d}\right) \in \mathbb{Z}^{d}$ with $x_{1}<0$ and any $\mathbf{a} \in E^{\mathbb{Z}^{d}}$.

Lemma 2. Let $k$ be a positive number. Then there exist positive constants $C_{i}, i=1,2$ such that

$$
P_{(x, \mathbf{a})}\left[X_{t}^{1} \leq C_{1} \quad \text { for some } \quad t>0\right] \leq \frac{C_{2}}{x_{1}^{k}}
$$

for any $x=\left(x_{1}, \ldots, x_{d}\right)$ with $x_{1}>C_{1}$ and any $\mathbf{a} \in E^{\mathbb{Z}^{d}}$. 
Proof of Lemma 1. For $x=\left(x_{1}, \ldots, x_{d}\right)$, set $V_{0}(x, \mathbf{a})=x_{1}+\varphi_{0}(x, \mathbf{a})$, where $\varphi_{0}$ will be determined later on. Assuming $\varphi_{0}$ belongs to the domain of $G$, compute

$$
\begin{aligned}
\Delta V_{0}(x, \mathbf{a}) & =L V_{0}(x, \mathbf{a})+G V_{0}(x, \mathbf{a}) \\
& =\lambda_{+}^{1,0}-\lambda_{-}^{1,0}+\bar{\lambda}_{+}^{1}(a(x))-\bar{\lambda}_{-}^{1}(a(x))+L \varphi_{0}(x, \mathbf{a})+G \varphi_{0}(x, \mathbf{a}),
\end{aligned}
$$

where $\bar{\lambda}_{ \pm}^{1}(a)=\lambda_{ \pm}^{1}(a)-\lambda_{ \pm}^{1,0}$.

Since $\int_{E} \bar{\lambda}_{ \pm}^{1}(a) \mu(d a)=0$,

$$
\varphi_{ \pm}(x, \mathbf{a})=-\int_{0}^{\infty} E_{a(x)}\left[\bar{\lambda}_{ \pm}^{1}\left(a_{t}(x)\right)\right] d t
$$

is well defined. Let $\varphi_{0}(x, \mathbf{a})=\varphi_{+}(x, \mathbf{a})-\varphi_{-}(x, \mathbf{a})$. Obviously

$$
G \varphi_{0}(x, \mathbf{a})+\bar{\lambda}_{+}^{1}(a(x))-\bar{\lambda}_{-}^{1}(a(x))=0 .
$$

From (2),

$$
\left|\varphi_{ \pm}(x, \mathbf{a})\right| \leq \frac{M_{0}}{\alpha}
$$

where $M_{0}=\max \left\{\sum_{a \in E}\left|\bar{\lambda}_{+}^{i}(a)\right|, \sum_{a \in E}\left|\bar{\lambda}_{-}^{i}(a)\right| ; i=1, \ldots, d\right\}$.

Hence

$$
\left|L \varphi_{0}(x, \mathbf{a})\right| \leq \frac{8 M_{0} M_{1} d}{\alpha},
$$

with $M_{1}=\max \left\{\lambda_{ \pm}^{1}(a), \ldots, \lambda_{ \pm}^{d}(a) ; a \in E\right\}$.

Thus if $\alpha>\beta_{0}^{d}=\frac{8 M_{0} M_{1} d}{\lambda_{+}^{1,0}-\lambda_{-}^{1,0}}$, then $\Delta V_{0}(x, \mathbf{a}) \geq 0$ for any $(x, \mathbf{a}) \in \mathbb{Z}^{d} \times E^{\mathbb{Z}^{d}}$.

Define stopping times by

$$
\sigma^{0}=\inf \left\{t>0 ; X_{t}^{1} \geq 0\right\}
$$

and

$$
\tau^{-N}=\inf \left\{t>0 ; X_{t}^{1} \leq-N\right\}, \quad N \geq 1 .
$$

By Dynkin's formula, we get

$$
E_{(x, \mathbf{a})}\left[V_{0}\left(X_{\tau^{-N} \wedge \sigma^{0}}, \mathbf{a}_{\tau^{-N} \wedge \sigma^{0}}\right)\right] \geq V_{0}(x, \mathbf{a})
$$

for any $x=\left(x_{1}, \ldots, x_{d}\right)$ with $x_{1} \in(-N, 0)$ and any $\mathbf{a} \in E^{\mathbb{Z}^{d}}$. 
Therefore

$$
\left(-N+\frac{2 M_{0}}{\alpha}\right) P_{(x, \mathbf{a})}\left(\tau^{-N}<\sigma^{0}\right)+\frac{2 M_{0}}{\alpha} P_{(x, \mathbf{a})}\left(\tau^{-N}>\sigma^{0}\right) \geq V_{0}(x, \mathbf{a})
$$

for any $(x, \mathbf{a})$ with $x_{1} \in(-N,-1]$. Letting $N \uparrow+\infty$, we obtain

$$
\lim _{N \rightarrow+\infty} P_{(x, \mathbf{a})}\left(\tau^{-N}<\sigma^{0}\right)=0
$$

which implies $P_{(x, \mathbf{a})}\left(X_{t}^{1}=0\right.$ for some $\left.t>0\right)=1$ for $x_{1}<0$.

Proof of Lemma 2. For a given positive constant $k$, set $g_{1}(x)=\left|x_{1}\right|^{-k}$ and

$$
V_{1}(x)=g_{1}(x)+g_{1,+}^{1}(x) \varphi_{+}(x, \mathbf{a})+g_{1,-}^{1}(x) \varphi_{-}(x, \mathbf{a}),
$$

where $g_{1,+}^{1}(x)=g_{1}\left(x+e_{1}\right)-g_{1}(x), g_{1,-}^{1}(x)=g_{1}\left(x-e_{1}\right)-g_{1}(x)$ and $\varphi_{ \pm}(x, \mathbf{a})$ are the ones in the proof of Lemma 1.

Then it is easy to see;

$$
\mathcal{E}_{0} \equiv \inf _{\substack{(x, \mathbf{a}) \\ x_{1}=C_{1}}} V_{1}(x, \mathbf{a})>0 \quad \text { and } \quad \Delta V_{1}(x, \mathbf{a}) \leq 0, \quad \text { if } \quad x_{1} \geq C_{1}
$$

for some positive integer $C_{1}$ and

$$
\lim _{x_{1} \rightarrow+\infty} V_{1}(x, \mathbf{a})=0 .
$$

Let

$$
\begin{aligned}
\sigma^{N} & =\inf \left\{t>0 ; X_{t}^{1} \geq N\right\}, \quad N>C_{1}, \\
\tau^{C_{1}} & =\inf \left\{t>0 ; X_{t}^{1} \leq C_{1}\right\} .
\end{aligned}
$$

Apply Dynkin's formula to obtain

$$
E_{(x, \mathbf{a})}\left[V_{1}\left(X_{\sigma^{N} \wedge \tau^{C_{1}}}, \mathbf{a}_{\sigma^{N} \wedge \tau^{C_{1}}}\right)\right] \leq V_{1}(x, \mathbf{a})
$$

for any $(x, \mathbf{a})$ with $x_{1} \in\left(C_{1}, N\right)$.

Letting $N \uparrow \infty$ in (7), we get

$$
P_{(x, \mathbf{a})}\left(\tau^{C_{1}}<+\infty\right) \leq \frac{V_{1}(x, \mathbf{a})}{\mathcal{E}_{0}} \quad \text { for } \quad x_{1}>C_{1} .
$$

Since $V_{1}(x, \mathbf{a})=O\left(\left|x_{1}\right|^{-k}\right)$, the proof of Lemma 2 is complete. 
In order to prove Theorem 1, we first note that there exist a point $\left(x_{1}^{0}, \mathbf{a}_{1}^{0}\right) \in \mathbb{Z}^{d} \times E^{\mathbb{Z}^{d}}$ and an integer $m_{1}$ such that

$$
P_{\left(x_{1}^{0}, \mathbf{a}_{1}^{0}\right)}\left(X_{t}^{1}=m_{1} \quad \text { i.o. as } \quad t \uparrow+\infty\right)>0
$$

if the assertion in Theorem 1 is false.

We can assume $m_{1}=C_{1}$ without loss of generality. Choose an integer $\rho_{0}>C_{1}$ such that $\frac{C_{2}}{\rho_{0}^{k}}<1$.

Define a sequence of stopping times as follows;

$$
\begin{gathered}
\tau_{1}^{C_{1}}=\inf \left\{t \geq 0 ; X_{t}^{1} \leq C_{1}\right\} \\
\sigma_{1}^{\rho_{0}}=\inf \left\{t \geq \tau_{1}^{C_{1}} ; X_{t}^{1} \geq \rho_{0}\right\} \\
\cdots \cdots \\
\tau_{n}^{C_{1}}=\inf \left\{t \geq \sigma_{n-1}^{\rho_{0}} ; X_{t}^{1} \leq C_{1}\right\} \\
\sigma_{n}^{\rho_{0}}=\inf \left\{t \geq \tau_{n}^{C_{1}} ; X_{t}^{1} \geq \rho_{0}\right\}
\end{gathered}
$$

Note that $\sigma_{n}^{\rho_{0}}$ is finite with probability one for all $n$ from Lemma 1 . We want to estimate

$$
P_{\left(x_{1}^{0}, \mathbf{a}_{1}^{0}\right)}\left(\tau_{n}^{C_{1}}<+\infty\right)=P_{\left(x_{1}^{0}, \mathbf{a}_{1}^{0}\right)}\left[\tau_{1}^{C_{1}}<\sigma_{1}^{\rho_{0}}<\cdots<\sigma_{n-1}^{\rho_{0}}<\tau_{n}^{C_{1}}\right] .
$$

By repeated use of the strong Markov property, the right-hand side is equal to

$$
\begin{aligned}
& E_{\left(x_{1}^{0}, \mathbf{a}_{1}^{0}\right)}\left[\tau_{1}^{C_{1}}<+\infty, E_{\left(X_{\tau_{1} C_{1}}, \mathbf{a}_{\tau_{1} C_{1}}\right)}\left[\tau_{2}^{C_{1}}<+\infty, E_{\left(X_{\tau_{2}} C_{1}, \mathbf{a}_{\tau_{2}} C_{1}\right)}[\ldots\right.\right. \\
& \cdots\left[\tau_{n-1}^{C_{1}}<+\infty, E_{\left(X_{\tau_{n-1} C_{1}}, \mathbf{a}_{\tau_{n-1} C_{1}}\right)}\left[P_{\left(X_{\sigma_{n-1} \rho_{0}}, \mathbf{a}_{\sigma_{n-1} \rho_{0}}\right)}\left[\tau_{n}^{C_{1}}<+\infty\right] \cdots\right] .\right.
\end{aligned}
$$

By Lemma 2, we get

$$
P_{\left(x_{1}^{0}, \mathbf{a}_{1}^{0}\right)}\left(\tau_{n}^{C_{1}}<+\infty\right) \leq\left(\frac{C_{2}}{\rho_{0}^{k}}\right)^{n-1} P_{\left(x_{1}^{0}, \mathbf{a}_{1}^{0}\right)}\left[\tau_{1}^{C_{1}}<+\infty\right]
$$

Hence $P_{\left(x_{1}^{0}, \mathbf{a}_{1}^{0}\right)}\left(X_{t}^{1}=m_{1}\right.$ i.o. as $\left.t \uparrow+\infty\right)=0$. Thus the assertion of Theorem 1 is valid.

[2] Proof of Theorem 2. The basic idea is the same as in the proof of Theorem 1. To prove Theorem 2 , we shall make use of a function $V_{2}(x, \mathbf{a})$, 
$(x, \mathbf{a}) \in \mathbb{Z}^{1} \times E^{\mathbb{Z}^{1}}$ such that

$$
\lim _{|x| \rightarrow+\infty} \inf _{\mathbf{a}} V_{2}(x, \mathbf{a})=+\infty
$$

$$
\Delta V_{2}(x, \mathbf{a}) \leq 0 \quad \text { for any } \quad|x| \geq C_{3} \quad \text { and } \quad \mathbf{a} \in E^{\mathbb{Z}^{1}}
$$

with some positive constant $C_{3}$.

Set $g_{2}(x)=\log |x|$ and $g_{2}^{1}(x)=g_{2}(x+1)-g_{2}(x-1)$. A function $V_{2}$ as above will be constructed in a form of

$$
V_{2}(x, \mathbf{a})=g_{2}(x)+g_{2}^{1}(x) \sum_{n=0}^{\infty} \varphi_{n}(x, \mathbf{a}) .
$$

For this $V_{2}$, we first perform a heuristic computation.

$$
\Delta V_{2}(x, \mathbf{a})=L g_{2}(x, \mathbf{a})+\sum_{n=0}^{\infty} L\left(g_{2}^{1} \varphi_{n}\right)(x, \mathbf{a})+\sum_{n=0}^{\infty} g_{2}^{1}(x) G \varphi_{n}(x, \mathbf{a})
$$

First of all,

$$
L g_{2}(x, \mathbf{a})=-\frac{\lambda^{1,0}}{x^{2}}+\bar{\lambda}_{+}^{1}(a(x)) g_{2}^{1}(x)+O\left(x^{-3}\right)
$$

and

$$
\begin{aligned}
L\left(g_{2}^{1} \varphi_{n}\right)(x, \mathbf{a})= & \lambda_{+}^{1}(a(x))\left(g_{2}^{1}(x+1) \varphi_{n}(x+1, \mathbf{a})-g_{2}^{1}(x) \varphi_{n}(x, \mathbf{a})\right) \\
& +\lambda_{-}^{1}(a(x))\left(g_{2}^{1}(x-1) \varphi_{n}(x-1, \mathbf{a})-g_{2}^{1}(x) \varphi_{n}(x, \mathbf{a})\right) \\
= & L \varphi_{n}(x, \mathbf{a})+P_{n}(x, \mathbf{a})
\end{aligned}
$$

where

$$
\begin{aligned}
P_{n}(x, \mathbf{a})= & \lambda_{+}^{1}(a(x)) \varphi_{n}(x+1, \mathbf{a})\left(g_{2}^{1}(x+1)-g_{2}^{1}(x)\right) \\
& +\lambda_{-}^{1}(a(x)) \varphi_{n}(x-1, \mathbf{a})\left(g_{2}^{1}(x-1)-g_{2}^{1}(x)\right) .
\end{aligned}
$$

It is easily seen that

$$
\left|P_{n}(x, \mathbf{a})\right| \leq 2 \max \left(p_{1}, q_{1}\right) \sup _{(x, \mathbf{a})}\left|\varphi_{n}(x, \mathbf{a})\right| \cdot\left(\frac{2}{x^{2}}+O\left(x^{-3}\right)\right) .
$$

Thus it follows formally that

$$
\begin{aligned}
\Delta V_{2}(x, \mathbf{a}) & =-\frac{\lambda^{1,0}}{x^{2}}+O\left(x^{-3}\right)+g_{2}^{1}(x)\left\{\bar{\lambda}_{+}^{1}(a(x))+G \varphi_{0}(x, \mathbf{a})\right\} \\
& +g_{2}^{1}(x) \sum_{n=0}^{\infty}\left\{L \varphi_{n}(x, \mathbf{a})+G \varphi_{n+1}(x, \mathbf{a})\right\}+\sum_{n=0}^{\infty} P_{n}(x, \mathbf{a}) .
\end{aligned}
$$


In order for $V_{2}$ to satisfy the conditions (8) and (9), we shall construct an infinite sequence of $\left\{\varphi_{n}\right\}$ such that

$$
\left\{\begin{array}{l}
\bar{\lambda}_{+}^{1}(a(x))+G \varphi_{0}(x, \mathbf{a})=0 \\
L \varphi_{n}(x, \mathbf{a})+G \varphi_{n+1}(x, \mathbf{a})=0, \quad n \geq 0
\end{array}\right.
$$

and $\left\{\varphi_{n}\right\}$ converges to zero sufficiently fast. Thus the following lemma is a key to the proof of Theorem 2.

LEMma 3. For the equations (11), there exists a system of solutions $\left\{\varphi_{n}, n \geq 0\right\}$ such that

$$
\left|\varphi_{n}(x, \mathbf{a})\right| \leq \frac{\left(p_{1}+q_{1}\right)^{n+1} \cdot 5^{n}}{\alpha^{n+1}}, \quad n \geq 0 .
$$

Before proceeding to the proof of Lemma, we give

Sublemma 1. Let $m$ be a positive integer and $x_{1}, \ldots, x_{m}$ be $m$-distinct points in $\mathbb{Z}^{1}$. Set

$$
f(\mathbf{a})=\prod_{i=1}^{m} \bar{\lambda}_{j_{i}}^{1}\left(a\left(x_{i}\right)\right), \quad j_{i}=+ \text { or }-.
$$

Then $E_{\mathbf{a}}\left[f\left(\mathbf{a}_{t}\right)\right]=e^{-m \alpha t} f(\mathbf{a})$.

Proof of Lemma 3. Since $\int_{E} \bar{\lambda}_{+}^{1}(a) \mu(d a)=0$, the first equation in (11) has a solution $\varphi_{0}$ given by

$$
\begin{aligned}
\varphi_{0}(x, \mathbf{a}) & =-\int_{0}^{\infty} E_{a(x)}\left[\bar{\lambda}_{+}^{1}\left(a_{t}(x)\right)\right] d t=-\frac{1}{\alpha} \bar{\lambda}_{+}^{1}(a(x)) \\
& =-\frac{1}{2 \alpha}\left\{\bar{\lambda}_{+}^{1}(a(x))-\bar{\lambda}_{-}^{1}(a(x))\right\} .
\end{aligned}
$$

Based on the following claims, we can construct $\left\{\varphi_{n}\right\}_{n \geq 1}$ by induction.

Claim 1. Let $\varphi(x, \mathbf{a})$ be a polynomial of $\bar{\lambda}_{+}^{1}(a(x+\cdot)), \bar{\lambda}_{-}^{1}(a(x+\cdot))$. Suppose there exist two polynomials $\varphi_{+}(x, \mathbf{a})$ and $\varphi_{-}(x, \mathbf{a})$ of $\bar{\lambda}_{+}^{1}(a(x+\cdot))$ and $\bar{\lambda}_{-}^{1}(a(x+\cdot))$ such that

(i) $\varphi(x, \mathbf{a})=\varphi_{+}(x, \mathbf{a})-\varphi_{-}(x, \mathbf{a})$

(ii) by replacement between $\bar{\lambda}_{+}(a(x+p))$ and $\bar{\lambda}_{-}(a(x-p)) \quad(p \in \mathbb{Z})$, we get $\varphi_{-}(x, \mathbf{a})$ from $\varphi_{+}(x, \mathbf{a})$. 
Then

$$
\int_{E^{\mathbb{Z}^{1}}} L \varphi(x, \mathbf{a}) \boldsymbol{\mu}(d \mathbf{a})=0
$$

and hence

$$
\tilde{\varphi}(x, \mathbf{a})=-\int_{0}^{\infty} E_{\mathbf{a}}\left[L \varphi\left(x, \mathbf{a}_{t}\right)\right] d t
$$

is well defined. $\tilde{\varphi}(x, \mathbf{a})$ is also a polynomial of $\bar{\lambda}_{+}^{1}(a(x+\cdot))$ and $\bar{\lambda}_{-}^{1}(a(x+\cdot))$ and is decomposed into a difference of two polynomials as in (i) and (ii).

Claim 2. For a polynomial $\psi$ of $\bar{\lambda}_{ \pm}^{1}(a(x+\cdot))$ as

$$
\psi(x, \mathbf{a})=\sum c_{r_{1}, \ldots, r_{t} ; s_{1}, \ldots, s_{u}} \prod_{i=1}^{t} \bar{\lambda}_{+}^{1}\left(a\left(x+r_{i}\right)\right) \prod_{j=1}^{u} \bar{\lambda}_{-}^{1}\left(a\left(x+s_{j}\right)\right)
$$

where integers $r_{1}, \ldots, r_{t}, s_{1}, \ldots, s_{u}$ are distinct each other and summation is taken over distinct sets $\left\{r_{1}, \ldots, r_{t}, s_{1}, \ldots, s_{u}\right\}$, define its norm by

$$
\|\psi\|=\sum\left|c_{r_{1}, \ldots, r_{t} ; s_{1}, \ldots, s_{u}}\right| \cdot \frac{\left|p_{1}-q_{1}\right|^{t+u}}{2^{t+u}} .
$$

Then for $\varphi$ and $\tilde{\varphi}$ in Claim 1, we have

$$
\|\tilde{\varphi}\| \leq \frac{5}{\alpha}\left(p_{1}+q_{1}\right)\|\varphi\|
$$

Proof of Claim 1. First note that the assumptions (i) and (ii) together with the definition of $L$ imply that $L \varphi_{+}$and $L \varphi_{-}$are interchangeable as in (ii). Indeed the following replacement enables $L \varphi_{+}$and $L \varphi_{-}$to interchange each other;

$$
\begin{aligned}
\bar{\lambda}_{+}^{1}(a(x)) & \prod_{j=1}^{l_{1}} \bar{\lambda}_{+}^{1}\left(a\left(x+r_{j}+1\right)\right) \prod_{k=1}^{l_{2}} \bar{\lambda}_{-}^{1}\left(a\left(x+s_{k}+1\right)\right) \\
& \longleftrightarrow \bar{\lambda}_{-}^{1}(a(x)) \prod_{j=1}^{l_{1}} \bar{\lambda}_{-}^{1}\left(a\left(x-r_{j}-1\right)\right) \prod_{k=1}^{l_{2}} \bar{\lambda}_{+}^{1}\left(a\left(x-s_{k}-1\right)\right) .
\end{aligned}
$$

Consequently, taking the configuration of environment into consideration, we see that if any constant terms appear in $L \varphi_{+}(x, \mathbf{a})$ and $L \varphi_{-}(x, \mathbf{a})$, they are the same. Thus it follows that

$$
\begin{aligned}
& \int_{E_{\mathbb{Z}^{1}}} L \varphi(x, \mathbf{a}) \boldsymbol{\mu}(d \mathbf{a}) \\
= & \int_{E^{\mathbb{Z}^{1}}}\left\{L \varphi_{+}(x, \mathbf{a})-L \varphi_{-}(x, \mathbf{a})\right\} \boldsymbol{\mu}(d \mathbf{a})=0
\end{aligned}
$$


Hence from Sublemma 1,

$$
\tilde{\varphi}(x, \mathbf{a})=-\int_{0}^{\infty} E_{\mathbf{a}}\left[L \varphi\left(x, \mathbf{a}_{t}\right)\right] d t
$$

is well defined. Now set

$$
\tilde{\varphi}_{ \pm}(x, \mathbf{a})=-\int_{0}^{\infty} E_{\mathbf{a}}\left[L \varphi_{ \pm}\left(x, \mathbf{a}_{t}\right)-\int_{E^{\mathbb{Z}^{1}}} L \varphi_{ \pm}(x, \mathbf{a}) \boldsymbol{\mu}(d \mathbf{a})\right] d t
$$

Obviously $\tilde{\varphi}(x, \mathbf{a})=\tilde{\varphi}_{+}(x, \mathbf{a})-\tilde{\varphi}_{-}(x, \mathbf{a})$. Since $L \varphi_{+}$and $L \varphi_{-}$are interchangeable, so are $\tilde{\varphi}_{+}$and $\tilde{\varphi}_{-}$by virtue of Sublemma 1.

Proof of Claim 2. Note that

$$
\begin{aligned}
L \varphi(x, \mathbf{a})= & \bar{\lambda}_{+}^{1}(a(x)) \varphi(x+1, \mathbf{a})+\bar{\lambda}_{-}^{1}(a(x)) \varphi(x-1, \mathbf{a}) \\
& +\lambda^{1,0} \varphi(x+1, \mathbf{a})+\lambda^{1,0} \varphi(x-1, \mathbf{a})-2 \lambda^{1,0} \varphi(x, \mathbf{a}) .
\end{aligned}
$$

Combining this with Sublemma 1, it is easy to see that (13) holds.

We now complete the proof of Lemma 3.

The existence of $\left\{\varphi_{n}\right\}$ follows from repeated use of Claim 1 started with $\varphi_{0}(x, \mathbf{a})=-\frac{1}{2 \alpha}\left\{\bar{\lambda}_{+}^{1}(a(x))-\bar{\lambda}_{-}^{1}(a(x))\right\}$. As for the upper bounds, we apply (13) with $\left\|\varphi_{0}\right\| \leq \frac{p_{1}+q_{1}}{\alpha}$.

Back to the proof of Theorem 2, we first assume $\frac{5\left(p_{1}+q_{1}\right)}{\alpha}<1$. Then $\sum_{n=0}^{\infty} \varphi_{n}(x, \mathbf{a})$ is convergent uniformly in $(x, \mathbf{a})$.

Since $\left|P_{n}(x, \mathbf{a})\right| \leq 4 \max \left(p_{1}, q_{1}\right) \cdot \sup \left|\varphi_{n}(x, \mathbf{a})\right| \cdot\left(x^{-2}+O\left(x^{-3}\right)\right)$, the conditions (8), (9) are fulfilled provided that $\alpha>13\left(p_{1}+q_{1}\right)$. Under this condition, $\sum_{n=0}^{\infty} \varphi_{n}(x, \mathbf{a})$ belongs to the domain of $G$ (See Liggett [3] for its definition.), since $\varphi_{n}(x, \mathbf{a})$ depends only on at most $(2 n+1)$ distinct points in $\mathbb{Z}^{1}$. This makes the computation for $\Delta V_{2}$ rigorous.

To complete the proof of Theorem 2, we introduce

$$
\begin{aligned}
\tau^{C_{3}} & =\inf \left\{t>0 ;\left|X_{t}\right| \leq C_{3}\right\} \\
\sigma^{N} & =\inf \left\{t>0 ;\left|X_{t}\right| \geq N\right\}, \quad N>C_{3} .
\end{aligned}
$$

Apply Dynkin's formula to get

$$
E_{(x, \mathbf{a})} V_{2}\left(X_{\tau_{C_{3}} \wedge \sigma_{N}}, \mathbf{a}_{\tau_{C_{3}} \wedge \sigma_{N}}\right) \leq V_{2}(x, \mathbf{a})
$$


for $C_{3}<|x|<N$. Therefore for $C_{3}<|x|<N$,

$$
\begin{aligned}
& \left\{\inf _{\substack{|x| \geq N \\
\mathbf{a} \in E^{1}}} V_{2}(x, \mathbf{a})\right\} \cdot P_{(x, \mathbf{a})}\left(\tau_{C_{3}}>\sigma_{N}\right)+\left\{\inf _{\substack{|x| \leq C_{3} \\
\mathbf{a} \in E^{1}}} V_{2}(x, \mathbf{a})\right\} \cdot P_{(x, \mathbf{a})}\left(\tau_{C_{3}}<\sigma_{N}\right) \\
& \leq V_{2}(x, \mathbf{a}) .
\end{aligned}
$$

Notice that $P_{(x, \mathbf{a})}\left(\tau_{C_{3}}>\sigma_{N}\right)+P_{(x, \mathbf{a})}\left(\tau_{C_{3}}<\sigma_{N}\right)=1$. Letting $N \uparrow \infty$, we obtain

$$
P_{(x, \mathbf{a})}\left(\tau_{C_{3}}<+\infty\right)=1 .
$$

Thus Theorem 2 is valid.

[3] Proof of Theorem 3. We shall find a function $V_{3}(x, \mathbf{a}),(x, \mathbf{a}) \in$ $\mathbb{Z}^{d} \times E^{\mathbb{Z}^{d}}$ such that

$$
V_{3}(x, \mathbf{a}) \geq 0 \quad \text { and } \quad \Delta V_{3}(x, \mathbf{a}) \leq 0
$$

for any $|x| \geq C_{4}$ and $\mathbf{a} \in E^{\mathbb{Z}^{d}}$ with a positive constant $C_{4}$ and

$$
\lim _{|x| \rightarrow+\infty} V_{3}(x, \mathbf{a})=0
$$

For a given constant $k \in(0, d-2)$, set $g_{3}(x)=r^{-k}, r^{2}=\frac{x_{1}^{2}}{\lambda^{1,0}}+\frac{x_{2}^{2}}{\lambda^{2,0}}+$ $\cdots+\frac{x_{d}^{2}}{\lambda^{d, 0}}, x=\left(x_{1}, \ldots, x_{d}\right)$ and $g_{3}^{i}(x)=g_{3}\left(x+e_{i}\right)-g_{3}\left(x-e_{i}\right)$. A Lyapunov function $V_{3}$ as above will be constructed in the following form,

$$
V_{3}(x, \mathbf{a})=g_{3}(x)+\sum_{i=1}^{d} g_{3}^{i}(x) \sum_{n=0}^{\infty} \varphi_{n}^{d, i}(x, \mathbf{a}) .
$$

By a heuristic computation,

$$
\Delta V_{3}(x, \mathbf{a})=L g_{3}(x, \mathbf{a})+\sum_{i=1}^{d} g_{3}^{i}(x) \sum_{n=0}^{\infty} G \varphi_{n}^{d, i}(x, \mathbf{a})+\sum_{i=1}^{d} \sum_{n=0}^{\infty} L\left(g_{3}^{i} \varphi_{n}^{d, i}\right)(x, \mathbf{a})
$$

On the other hand,

$$
L g_{3}(x, \mathbf{a})=-\frac{k(d-k-2)}{r^{k+2}}+O\left(r^{-k-3}\right)+\sum_{i=1}^{d} \bar{\lambda}_{+}^{i}(a(x)) g_{3}^{i}(x)
$$

with $\bar{\lambda}_{+}^{i}(a)=\lambda_{+}^{i}(a)-\lambda^{i, 0}$ and

$$
L\left(g_{3}^{i} \varphi_{n}^{d, i}\right)(x, \mathbf{a})=L \varphi_{n}^{d, i}(x, \mathbf{a})+Q_{n}^{i}(x, \mathbf{a})
$$


where

$$
\begin{aligned}
Q_{n}^{i}(x, \mathbf{a})=\sum_{j=1}^{d} & \left\{\lambda_{+}^{j}(a(x))\left(g_{3}^{i}\left(x+e_{j}\right)-g_{3}^{i}(x)\right) \varphi_{n}^{d, i}\left(x+e_{j}, \mathbf{a}\right)\right. \\
& \left.+\lambda_{-}^{j}(a(x))\left(g_{3}^{i}\left(x-e_{j}\right)-g_{3}^{i}(x)\right) \varphi_{n}^{d, i}\left(x-e_{j}, \mathbf{a}\right)\right\} .
\end{aligned}
$$

Note that

$$
\left|Q_{n}^{i}(x, \mathbf{a})\right| \leq \sup _{x, \mathbf{a}, i}\left|\varphi_{n}^{d, i}(x, \mathbf{a})\right| \cdot\left(\frac{C_{5}}{r^{k+2}}+O\left(r^{-k-3}\right)\right)
$$

with a positive constant $C_{5}$.

Thus we have

$$
\begin{aligned}
\Delta V_{3}(x, \mathbf{a}) & =-\frac{k(d-k-2)}{r^{k+2}}+O\left(r^{-k-3}\right) \\
& +\sum_{i=1}^{d} g_{3}^{i}(x)\left\{\bar{\lambda}_{+}^{i}(a(x))+G \varphi_{0}^{d, i}(x, \mathbf{a})\right\} \\
& +\sum_{i=1}^{d} g_{3}^{i}(x) \sum_{n=0}^{\infty}\left\{L \varphi_{n}^{d, i}(x, \mathbf{a})+G \varphi_{n+1}^{d, i}(x, \mathbf{a})\right\} \\
& +\sum_{i=1}^{d} \sum_{n=0}^{\infty} Q_{n}^{i}(x, \mathbf{a})
\end{aligned}
$$

As in the proof of Theorem 2, the following lemma is a key to the proof of Theorem 3.

LEMMA 4. The following d-systems of equations;

$$
\left\{\begin{array}{l}
\bar{\lambda}_{+}^{i}(a(x))+G \varphi_{0}^{d, i}(x, \mathbf{a})=0 \\
L \varphi_{n}^{d, i}(x, \mathbf{a})+G \varphi_{n+1}^{d, i}(x, \mathbf{a})=0, \quad n \geq 0 \quad \text { and } \quad i=1, \ldots, d
\end{array}\right.
$$

have solutions $\left\{\varphi_{n}^{d, i}\right\}_{n=0,1, \ldots}^{i=1, \ldots, d}$ such that

$$
\left|\varphi_{n}^{d, i}(x, \mathbf{a})\right| \leq \frac{\left(p_{i}+q_{i}\right)\left\{\max \left\{p_{j}+q_{j} ; j=1, \ldots, d\right\}\right\}^{n}(4 d+1)^{n}}{\alpha^{n+1}}
$$

For the proof of Lemma 3, we need the next claims. 
Claim 3. Let $\varphi(x, \mathbf{a})$ be a polynomial of $\bar{\lambda}_{ \pm}^{i}(a(x+\cdot)), i=1, \ldots, d$. Suppose there exist two polynomials $\varphi_{+}(x, \mathbf{a}), \varphi_{-}(x, \mathbf{a})$ of $\lambda_{ \pm}^{i}(a(x+\cdot))$, $i=1, \ldots, d$ such that

(i) $\varphi(x, \mathbf{a})=\varphi_{+}(x, \mathbf{a})-\varphi_{-}(x, \mathbf{a})$

(ii) by replacement between $\bar{\lambda}_{+}^{i}(a(x+p))$ and $\bar{\lambda}_{-}^{i}(a(x-p)) \quad\left(p \in \mathbb{Z}^{d}, i=\right.$ $1, \ldots, d)$ we get $\varphi_{-}(x, \mathbf{a})$ from $\varphi_{+}(x, \mathbf{a})$.

Then

$$
\int_{E^{\mathbb{Z}^{d}}} L \varphi(x, \mathbf{a}) \boldsymbol{\mu}(d \mathbf{a})=0
$$

and hence

$$
\tilde{\varphi}(x, \mathbf{a})=-\int_{0}^{\infty} E_{\mathbf{a}}\left[L \varphi\left(x, \mathbf{a}_{t}\right)\right] d t
$$

is well defined. $\tilde{\varphi}(x, \mathbf{a})$ is also a polynomial of $\lambda_{ \pm}^{i}(a(x+\cdot))$ and is decomposed into a difference of two polynomials as in (i) and (ii).

Claim 4. For a polynomial $\psi$ of $\bar{\lambda}_{ \pm}^{i}(a(x+\cdot)), i=1, \ldots, d$ as

$$
\begin{array}{r}
\psi(x, \mathbf{a})=\sum c_{r_{1}^{1}, \ldots, r_{t_{1}}^{1}, s_{1}^{1}, \ldots, s_{u_{1}}^{1} ; r_{1}^{2}, \ldots, r_{t_{2}}^{2}, s_{1}^{2}, \ldots, s_{u_{2}}^{2} ; \cdots ; r_{1}^{d}, \ldots, r_{t_{d}}^{d}, s_{1}^{d}, \ldots, s_{u_{d}}^{d}} \\
\times \prod_{j=1}^{d}\left(\prod_{i=1}^{t_{j}} \bar{\lambda}_{+}^{j}\left(a\left(x+r_{i}^{j}\right)\right) \prod_{k=1}^{u_{j}} \bar{\lambda}_{-}^{j}\left(a\left(x+s_{k}^{j}\right)\right)\right)
\end{array}
$$

where for each $j$, integers $r_{1}^{j}, \ldots, r_{t_{j}}^{j}, s_{1}^{j}, \ldots, s_{u_{j}}^{j}$ are distinct and summation is taken over distinct direct products $\left\{r_{1}^{1}, \ldots, r_{t_{1}}^{1}, s_{1}^{1}, \ldots, s_{u_{1}}^{1}\right\} \times$ $\left\{r_{1}^{2}, \ldots, r_{t_{2}}^{2}, s_{1}^{2}, \ldots, s_{u_{2}}^{2}\right\} \times \cdots \times\left\{r_{1}^{d}, \ldots, r_{t_{d}}^{d}, s_{1}^{d}, \ldots, s_{u_{d}}^{d}\right\}$,

define

$$
\|\psi\|=\sum\left|c_{r_{1}^{1}, \ldots, r_{t_{1}}^{1}, s_{1}^{1}, \ldots, s_{u_{1}}^{1} ; \cdots ; r_{1}^{d}, \ldots, r_{t_{d}}^{d}, s_{1}^{d}, \ldots, s_{u_{d}}^{d}}\right| \times \prod_{j=1}^{d} \frac{\left|p_{j}-q_{j}\right|^{t_{j}+u_{j}}}{2^{t_{j}+u_{j}}} .
$$

Then for $\varphi$ and $\tilde{\varphi}$ in Claim 3,

$$
\|\tilde{\varphi}\| \leq \frac{(4 d+1) \max \left\{p_{j}+q_{j} ; j=1, \ldots, d\right\}}{\alpha}\|\varphi\| .
$$


Since the proofs of Claim 3, 4 and Lemma 4 are similar to those of Claim 1, 2 and Lemma 3, they are omitted.

The rest of the proof of Theorem 3 is also worked out as in the proof of Theorem 1. Therefore we omit it, too.

[4] Proof of Theorem 4. As in the proof of Theorem 2, the crucial step is to construct a Lyapunov function $V_{4}(x, \mathbf{a}),(x, \mathbf{a}) \in \mathbb{Z}^{2} \times E^{\mathbb{Z}^{2}}$ such that

$$
\Delta V_{4}(x, \mathbf{a}) \leq 0 \quad \text { for } \quad|x| \geq C_{6} \quad \text { and } \quad \mathbf{a} \in E^{\mathbb{Z}^{2}}
$$

with a positive constant $C_{6}$ and

$$
\lim _{|x| \rightarrow \infty} \inf _{a \in E^{\mathbb{Z}^{2}}} V_{4}(x, \mathbf{a})=+\infty
$$

However we need a more elaborate argument for this case.

Putting $g_{4}(x)=\log ^{2}|x|=\log (\log |x|),|x|=\sqrt{x_{1}^{2}+x_{2}^{2}}$, a function $V_{4}$ as above will be constructed in the following form;

$$
\begin{aligned}
V_{4}(x, \mathbf{a})=g_{4}(x) & +g_{4}^{1}(x) \sum_{n=0}^{\infty} \psi_{n}^{1}(x, \mathbf{a})+g_{4}^{2}(x) \sum_{n=0}^{\infty} \psi_{n}^{2}(x, \mathbf{a}) \\
& +g_{4}^{1,1}(x) \sum_{n=0}^{\infty} \sum_{m=0}^{\infty} \psi_{n, m}^{1}(x, \mathbf{a}) \\
& +g_{4}^{1,2}(x) \sum_{n=0}^{\infty} \sum_{m=0}^{\infty} \psi_{n, m}^{2}(x, \mathbf{a})
\end{aligned}
$$

where

$$
\begin{array}{ll}
g_{4}^{1}(x)=g_{4}\left(x+e_{1}\right)-g_{4}\left(x-e_{1}\right), & g_{4}^{2}(x)=g_{4}\left(x+e_{2}\right)-g_{4}\left(x-e_{2}\right), \\
g_{4}^{1,1}(x)=\frac{2\left(x_{2}^{2}-x_{1}^{2}\right)}{|x|^{4} \log |x|}, & g_{4}^{1,2}(x)=-\frac{4 x_{1} x_{2}}{|x|^{4} \log |x|} .
\end{array}
$$


A heuristic computation shows,

$$
\begin{aligned}
\Delta V_{4}(x, \mathbf{a}) & =L g_{4}(x, \mathbf{a})+\sum_{n=0}^{\infty} g_{4}^{1}(x) G \psi_{n}^{1}(x, \mathbf{a})+\sum_{n=0}^{\infty} g_{4}^{2}(x) G \psi_{n}^{2}(x, \mathbf{a}) \\
& +\sum_{n=0}^{\infty} L\left(g_{4}^{1} \psi_{n}^{1}\right)(x, \mathbf{a})+\sum_{n=0}^{\infty} L\left(g_{4}^{2} \psi_{n}^{2}\right)(x, \mathbf{a}) \\
& +\sum_{n=0}^{\infty} \sum_{m=0}^{\infty} g_{4}^{1,1}(x) G \psi_{n, m}^{1}(x, \mathbf{a})+\sum_{n=0}^{\infty} \sum_{m=0}^{\infty} g_{4}^{1,2}(x) G \psi_{n, m}^{2}(x, \mathbf{a}) \\
& +\sum_{n=0}^{\infty} \sum_{m=0}^{\infty} L\left(g_{4}^{1,1} \psi_{n, m}^{1}\right)(x, \mathbf{a})+\sum_{n=0}^{\infty} \sum_{m=0}^{\infty} L\left(g_{4}^{1,2} \psi_{n, m}^{2}\right)(x, \mathbf{a}) .
\end{aligned}
$$

It is easy to see,

$$
L g_{4}(x, \mathbf{a})=L_{0} g_{4}(x, \mathbf{a})+L_{1} g_{4}(x, \mathbf{a})
$$

where

$$
\begin{aligned}
L_{0} g_{4}(x)= & \lambda^{0}\left\{\left(g_{4}\left(x+e_{1}\right)-g_{4}(x)\right)+\left(g_{4}\left(x-e_{1}\right)-g_{4}(x)\right)\right. \\
& \left.+\left(g_{4}\left(x+e_{2}\right)-g_{4}(x)\right)+\left(g_{4}\left(x-e_{2}\right)-g_{4}(x)\right)\right\} \\
= & -\frac{\lambda^{0}}{|x|^{2}(\log |x|)^{2}}+O\left(\frac{1}{|x|^{3} \log |x|}\right)
\end{aligned}
$$

and

$$
L_{1} g_{4}(x, \mathbf{a})=\bar{\lambda}_{+}^{1}(a(x)) g_{4}^{1}(x)+\bar{\lambda}_{+}^{2}(a(x)) g_{4}^{2}(x)
$$

with $\lambda^{0}=\lambda^{1,0}=\lambda^{2,0}$ and $\bar{\lambda}_{+}^{i}(a)=\lambda_{+}^{i}(a)-\lambda^{0}(i=1,2)$.

Furthermore,

$$
\begin{aligned}
L\left(g_{4}^{1} \psi_{n}^{1}\right)(x, \mathbf{a}) & =g_{4}^{1}(x) L \psi_{n}^{1}(x, \mathbf{a})+g_{4}^{1,1}(x) T_{1} \psi_{n}^{1}(x, \mathbf{a}) \\
& +g_{4}^{1,2}(x) T_{2} \psi_{n}^{1}(x, \mathbf{a})+E_{1}^{n}(x, \mathbf{a}), \\
L\left(g_{4}^{2} \psi_{n}^{2}\right)(x, \mathbf{a}) & =g_{4}^{2}(x) L \psi_{n}^{2}(x, \mathbf{a})+g_{4}^{1,2}(x) T_{1} \psi_{n}^{2}(x, \mathbf{a}) \\
& -g_{4}^{1,1}(x) T_{2} \psi_{n}^{2}(x, \mathbf{a})+E_{2}^{n}(x, \mathbf{a}), \\
L\left(g_{4}^{1,1} \psi_{n, m}^{1}\right)(x, \mathbf{a}) & =g_{4}^{1,1}(x) L \psi_{n, m}^{1}(x, \mathbf{a})+E_{3}^{n, m}(x, \mathbf{a}), \\
L\left(g_{4}^{1,2} \psi_{n, m}^{2}\right)(x, \mathbf{a}) & =g_{4}^{1,2}(x) L \psi_{n, m}^{2}(x, \mathbf{a})+E_{4}^{n, m}(x, \mathbf{a})
\end{aligned}
$$

where

$$
\begin{aligned}
& T_{1} \psi(x, \mathbf{a})=\lambda_{+}^{1}(a(x)) \psi\left(x+e_{1}, \mathbf{a}\right)-\lambda_{-}^{1}(a(x)) \psi\left(x-e_{1}, \mathbf{a}\right), \\
& T_{2} \psi(x, \mathbf{a})=\lambda_{+}^{2}(a(x)) \psi\left(x+e_{2}, \mathbf{a}\right)-\lambda_{-}^{2}(a(x)) \psi\left(x-e_{2}, \mathbf{a}\right),
\end{aligned}
$$


and the error terms satisfy,

$$
\begin{gathered}
\left|E_{1}^{n}(x, \mathbf{a})\right| \leq C_{7} \frac{\sup _{x, \mathbf{a}}\left|\psi_{n}^{1}\right|}{|x|^{2}(\log |x|)^{2}}, \\
\left|E_{2}^{n}(x, \mathbf{a})\right| \leq C_{7} \frac{\sup _{7}\left|\psi_{n}^{2}\right|}{|x|^{2}(\log |x|)^{2}}, \\
\left|E_{3}^{n, m}(x, \mathbf{a})\right| \leq C_{7} \frac{x, \mathbf{a}}{|x|^{3} \log |x|}, \\
\left|E_{4}^{n, m}(x, \mathbf{a})\right| \leq C_{7} \frac{\sup _{n}\left|\psi_{n, m}^{2}\right|}{|x|^{3} \log |x|},
\end{gathered}
$$

for $\mathbf{a} \in E^{\mathbb{Z}^{2}}$ and $|x| \geq 2$ with a positive constant $C_{7}$ independent of $n, m$. Thus we have shown,

$$
\begin{aligned}
& \Delta V_{4}(x, \mathbf{a})=L_{0} g_{4}(x)+g_{4}^{1}(x)\left(\bar{\lambda}_{+}^{1}(a(x))+G \psi_{0}^{1}(x, \mathbf{a})\right) \\
& +g_{4}^{2}(x)\left(\bar{\lambda}_{+}^{2}(a(x))+G \psi_{0}^{2}(x, \mathbf{a})\right)+g_{4}^{1}(x) \sum_{n=0}^{\infty}\left(L \psi_{n}^{1}(x, \mathbf{a})+G \psi_{n+1}^{1}(x, \mathbf{a})\right) \\
& +g_{4}^{2}(x) \sum_{n=0}^{\infty}\left(L \psi_{n}^{2}(x, \mathbf{a})+G \psi_{n+1}^{2}(x, \mathbf{a})\right) \\
& +g_{4}^{1,1}(x) \sum_{n=0}^{\infty}\left\{\left(T_{1} \psi_{n}^{1}(x, \mathbf{a})-T_{2} \psi_{n}^{2}(x, \mathbf{a})+G \psi_{n, 0}^{1}(x, \mathbf{a})\right)\right. \\
& \left.+\sum_{m=0}^{\infty}\left(L \psi_{n, m}^{1}(x, \mathbf{a})+G \psi_{n, m+1}^{1}(x, \mathbf{a})\right)\right\} \\
& +g_{4}^{1,2}(x) \sum_{n=0}^{\infty}\left\{\left(T_{2} \psi_{n}^{1}(x, \mathbf{a})+T_{1} \psi_{n}^{2}(x, \mathbf{a})+G \psi_{n, 0}^{2}(x, \mathbf{a})\right)\right. \\
& \left.+\sum_{m=0}^{\infty}\left(L \psi_{n, m}^{2}(x, \mathbf{a})+G \psi_{n, m+1}^{2}(x, \mathbf{a})\right)\right\} \\
& +\sum_{n=0}^{\infty}\left\{E_{1}^{n}(x, \mathbf{a})+E_{2}^{n}(x, \mathbf{a})+\sum_{m=0}^{\infty} E_{3}^{n, m}(x, \mathbf{a})+\sum_{m=0}^{\infty} E_{4}^{n, m}(x, \mathbf{a})\right\}
\end{aligned}
$$

To obtain a desired Lyapunov function in (17), (18), we first give, 
LEMMA 5. There exist two infinite sequences of solutions $\left\{\psi_{n}^{i}\right\}_{n \geq 0}^{i=1,2}$ for the following equations ; for $i=1,2$,

$$
\left\{\begin{array}{l}
\bar{\lambda}_{+}^{i}(a(x))+G \psi_{0}^{i}(x, \mathbf{a})=0 \\
L \psi_{n}^{i}(x, \mathbf{a})+G \psi_{n+1}^{i}(x, \mathbf{a})=0, \quad n \geq 0,
\end{array}\right.
$$

which have the following upper bounds,

$$
\left|\psi_{n}^{i}(x, \mathbf{a})\right| \leq \frac{9^{n}(p+q)^{n+1}}{\alpha^{n+1}}, \quad n \geq 0 .
$$

This lemma is a special case of Lemma 4.

For the solutions $\left\{\psi_{n}^{i}\right\}$ obtained in Lemma 5, we have

Lemma 6. For each $n \geq 0$, there exists an infinite sequence of solutions $\left\{\psi_{n, m}^{1}\right\}_{m \geq 0}$ for the equations;

$$
\left\{\begin{array}{l}
T_{1} \psi_{n}^{1}(x, \mathbf{a})-T_{2} \psi_{n}^{2}(x, \mathbf{a})+G \psi_{n, 0}^{1}(x, \mathbf{a})=0 \\
L \psi_{n, m}^{1}(x, \mathbf{a})+G \psi_{n, m+1}^{1}(x, \mathbf{a})=0, \quad m \geq 0
\end{array}\right.
$$

which have the following upper bounds,

$$
\left|\psi_{n, m}^{1}(x, \mathbf{a})\right| \leq \frac{4 \cdot 9^{m+n}(p+q)^{m+n+2}}{\alpha^{n+m+2}} .
$$

LEMMA 7. For each $n \geq 0$, there exists an infinite sequence of solutions $\left\{\psi_{n, m}^{2}\right\}_{m \geq 0}$ for the equations;

$$
\left\{\begin{array}{l}
T_{2} \psi_{n}^{1}(x, \mathbf{a})+T_{1} \psi_{n}^{2}(x, \mathbf{a})+G \psi_{n, 0}^{2}(x, \mathbf{a})=0 \\
L \psi_{n, m}^{2}(x, \mathbf{a})+G \psi_{n, m+1}^{2}(x, \mathbf{a})=0, \quad m \geq 0
\end{array}\right.
$$

which have the following upper bounds,

$$
\left|\psi_{n, m}^{2}(x, \mathbf{a})\right| \leq \frac{4 \cdot 9^{m+n}(p+q)^{m+n+2}}{\alpha^{n+m+2}} .
$$

For the proof of the above three lemmas, the following is also necessary.

Sublemma 2. For the Markov chain $a_{t}$ on $E$, we have

$$
E_{a_{0}}\left[\bar{\lambda}_{j_{1}}^{1}\left(a_{t}\right) \bar{\lambda}_{j_{2}}^{2}\left(a_{t}\right)\right]=e^{-\alpha t} \bar{\lambda}_{j_{1}}^{1}\left(a_{0}\right) \bar{\lambda}_{j_{2}}^{2}\left(a_{0}\right)
$$

with $j_{1}, j_{2}=+$ or - . 
Proof of Lemma 6. From the definitions of $T_{i}$ and $\psi_{n}^{i}(x, \mathbf{a}), i=1,2$, $n \geq 0$, we can easily see that $T_{1} \psi_{n}^{1}(x, \mathbf{a})$ and $T_{2} \psi_{n}^{2}(x, \mathbf{a})$ interchange each other through replacement;

$$
\left\{\begin{array}{l}
\bar{\lambda}_{+}^{1}\left(a\left(x+\alpha e_{1}+\beta e_{2}\right)\right) \longleftrightarrow \bar{\lambda}_{+}^{2}\left(a\left(x+\beta e_{1}+\alpha e_{2}\right)\right) \\
\bar{\lambda}_{-}^{1}\left(a\left(x+\alpha e_{1}+\beta e_{2}\right)\right) \longleftrightarrow \bar{\lambda}_{-}^{2}\left(a\left(x+\beta e_{1}+\alpha e_{2}\right)\right),
\end{array}\right.
$$

$\alpha, \beta \in \mathbb{Z}$. Taking this into account, the next claim implies that the assertion of Lemma 6 is valid.

Claim 5. Let $\varphi$ be a polynomial of $\bar{\lambda}_{ \pm}^{1}(a(x+\cdot)), \bar{\lambda}_{ \pm}^{2}(a(x+\cdot))$. Suppose there exist two polynomials $\varphi_{+}$and $\varphi_{-}$of $\bar{\lambda}_{ \pm}^{1}(a(x+\cdot)), \bar{\lambda}_{ \pm}^{2}(a(x+\cdot))$ such that

(i) $\varphi(x, \mathbf{a})=\varphi_{+}(x, \mathbf{a})-\varphi_{-}(x, \mathbf{a})$

(ii) by the replacement (19), we get $\varphi_{-}(x, \mathbf{a})$ from $\varphi_{+}(x, \mathbf{a})$.

Then

$$
\int_{E^{\mathbb{Z}^{2}}} L \varphi(x, \mathbf{a}) \boldsymbol{\mu}(d \mathbf{a})=0
$$

and hence

$$
\tilde{\varphi}(x, \mathbf{a})=-\int_{0}^{\infty} E_{\mathbf{a}}\left[L \varphi\left(x, \mathbf{a}_{t}\right)\right] d t
$$

is well defined. $\tilde{\varphi}(x, \mathbf{a})$ is also a polynomial as in (i), (ii). And we have for the above $\varphi, \tilde{\varphi}$,

$$
\|\tilde{\varphi}\| \leq \frac{9}{\alpha}(p+q)\|\varphi\|
$$

with respect to the same norm as in Claim 4.

The proof of Claim 5 and the rest of the proof of Lemma 6 are basically the same as before. Therefore we omit the details. However we only note that under the assumption (ii) in Claim $5, L \varphi_{+}$and $L \varphi_{-}$interchange each 
other through the following replacement;

$$
\begin{aligned}
& \lambda_{(-)}^{\lambda_{+}}(a(x)) \prod_{j_{1}=1}^{t_{1}} \bar{\lambda}_{+}^{1}\left(a\left(x+\left(\alpha_{j_{1}}^{1}{\underset{(-)}{+}}_{(-1)} e_{1}+\beta_{j_{1}}^{1} e_{2}\right)\right)\right. \\
& \times \prod_{j_{2}=1}^{t_{2}} \bar{\lambda}_{-}^{1}\left(a\left(x+\left(\alpha_{j_{2}}^{2} \underset{(-)}{+} 1\right) e_{1}+\beta_{j_{2}}^{2} e_{2}\right)\right) \\
& \times \prod_{j_{3}=1}^{t_{3}} \bar{\lambda}_{+}^{2}\left(a\left(x+\left(\alpha_{j_{3}}^{3} \underset{(-)}{+} 1\right) e_{1}+\beta_{j_{3}}^{3} e_{2}\right)\right) \\
& \times \prod_{j_{4}=1}^{t_{4}} \bar{\lambda}_{-}^{2}\left(a\left(x+\left(\alpha_{j_{4}}^{4} \underset{(-)}{+} 1\right) e_{1}+\beta_{j_{4}}^{4} e_{2}\right)\right) \\
& \lambda_{(-)}^{2}(a(x)) \prod_{j_{1}=1}^{t_{1}} \bar{\lambda}_{+}^{2}\left(a\left(x+\beta_{j_{1}}^{1} e_{1}+\left(\alpha_{j_{1}}^{1} \underset{(-)}{+} 1\right) e_{2}\right)\right) \\
& \times \prod_{j_{2}=1}^{t_{2}} \bar{\lambda}_{-}^{2}\left(a\left(x+\beta_{j_{2}}^{2} e_{1}+\left(\alpha_{j_{2}}^{2} \underset{(-)}{+} 1\right) e_{2}\right)\right) \\
& \times \prod_{j_{3}=1}^{t_{3}} \bar{\lambda}_{+}^{1}\left(a\left(x+\beta_{j_{3}}^{3} e_{1}+\left(\alpha_{j_{3}}^{3} \underset{(-)}{+} 1\right) e_{2}\right)\right) \\
& \times \prod_{j_{4}=1}^{t_{4}} \bar{\lambda}_{-1}^{1}\left(a\left(x+\beta_{j_{4}}^{4} e_{1}+\left(\alpha_{j_{4}}^{4} \underset{(-)}{+} 1\right) e_{2}\right)\right) .
\end{aligned}
$$

Proof of Lemma 7. From the definition of $\psi_{n}^{1}, \psi_{n}^{1}\left(x+e_{2}, \mathbf{a}\right)$ and $\psi_{n}^{1}(x-$ $\left.e_{2}, \mathbf{a}\right)$ interchange one another through replacement;

$$
\left\{\begin{array}{l}
\bar{\lambda}_{{ }_{+}}^{1}\left(a\left(x+\alpha e_{1}+\beta e_{2}\right)\right) \longleftrightarrow \bar{\lambda}_{{ }_{+}}^{1}\left(a\left(x+\alpha e_{1}-\beta e_{2}\right)\right) \\
\bar{\lambda}_{+}^{2}\left(a\left(x+\alpha e_{1}+\beta e_{2}\right)\right) \longleftrightarrow \bar{\lambda}_{-}^{2}\left(a\left(x+\alpha e_{1}-\beta e_{2}\right)\right),
\end{array}\right.
$$

so do $\lambda_{+}^{2}(a(x)) \psi_{n}^{1}\left(x+e_{2}, \mathbf{a}\right)$ and $\lambda_{-}^{2}(a(x)) \psi_{n}^{1}\left(x-e_{2}, \mathbf{a}\right)$.

Similarly we see that $\lambda_{+}^{1}(a(x)) \psi_{n}^{2}\left(x+e_{1}, \mathbf{a}\right)$ and $\lambda_{-}^{1}(a(x)) \psi_{n}^{2}\left(x-e_{1}, \mathbf{a}\right)$ interchange one another through replacement;

$$
\left\{\begin{array}{l}
\bar{\lambda}^{2}{ }_{+}^{+}\left(a\left(x+\alpha e_{1}+\beta e_{2}\right)\right) \longleftrightarrow \bar{\lambda}_{++}^{2}\left(a\left(x-\alpha e_{1}+\beta e_{2}\right)\right) \\
\bar{\lambda}_{+}^{1}\left(a\left(x+\alpha e_{1}+\beta e_{2}\right)\right) \longleftrightarrow \bar{\lambda}_{-}^{1}\left(a\left(x-\alpha e_{1}+\beta e_{2}\right)\right) .
\end{array}\right.
$$


This combined with the next claim implies that Lemma 7 is valid.

Claim 6. Let $\varphi$ be a polynomial of $\bar{\lambda}_{ \pm}^{1}(a(x+\cdot)), \bar{\lambda}_{ \pm}^{2}(a(x+\cdot))$. Suppose there exist two polynomials $\varphi_{+}$and $\varphi_{-}$of $\bar{\lambda}_{ \pm}^{1}(a(x+\cdot)), \bar{\lambda}_{ \pm}^{2}(a(x+\cdot))$ such that

(i) $\varphi(x, \mathbf{a})=\varphi_{+}(x, \mathbf{a})-\varphi_{-}(x, \mathbf{a})$

(ii) by the replacement (20), we get $\varphi_{-}(x, \mathbf{a})$ from $\varphi_{+}(x, \mathbf{a})$.

Then

$$
\int_{E^{\mathbb{Z}^{2}}} L \varphi(x, \mathbf{a}) \boldsymbol{\mu}(d \mathbf{a})=0
$$

hence

$$
\tilde{\varphi}(x, \mathbf{a})=-\int_{0}^{\infty} E_{\mathbf{a}}\left[L \varphi\left(x, \mathbf{a}_{t}\right)\right] d t
$$

is well defined. $\tilde{\varphi}(x, \mathbf{a})$ is also a polynomial as in (i), (ii). And we have for the above $\varphi, \tilde{\varphi}$,

$$
\|\tilde{\varphi}\| \leq \frac{9}{\alpha}(p+q)\|\varphi\|
$$

with respect to the same norm $\|\cdot\|$ as in Claim 2.

Note that all the assertions in Claim 6 hold under another replacement (21) instead of (20).

The proof of Claim 6 is also similar to that of Claim 1. Therefore the detail is omitted.

We now complete the proof of Theorem 4. First note that by what has been proved above, the Lyapunov function $V_{4}$ satisfies all the conditions (17), (18) and that $V_{4}(x, \cdot)$ belongs to the domain of $G$ for all sufficiently large $\alpha$.

Define

$$
\tau_{C_{6}}=\inf \left\{t>0 ;\left|X_{t}\right| \leq C_{6}\right\}
$$

and

$$
\sigma_{N}=\inf \left\{t>0 ;\left|X_{t}\right| \geq N\right\} \text {. }
$$

Apply Dynkin's formula to obtain,

$$
E_{(x, \mathbf{a})}\left[V_{4}\left(X_{\tau_{C_{6}} \wedge \sigma_{N}}, \mathbf{a}_{\tau_{C_{6}} \wedge \sigma_{N}}\right)\right] \leq V_{4}(x, \mathbf{a}) \quad \text { for } \quad C_{6}<|x|<N .
$$


Therefore

$P_{(x, \mathbf{a})}\left(\tau_{C_{6}}<\sigma_{N}\right) \inf _{\substack{\mathbf{a} \in E^{\mathbb{Z}^{2}} \\|x| \leq C_{6}}} V_{4}(x, \mathbf{a})+P_{(x, \mathbf{a})}\left(\tau_{C_{6}}>\sigma_{N}\right) \inf _{\substack{\mathbf{a} \in E^{\mathbb{Z}^{2}} \\|x| \geq N}} V_{4}(x, \mathbf{a}) \leq V_{4}(x, \mathbf{a})$.

Letting $N \rightarrow+\infty$, we get

$$
P_{(x, \mathbf{a})}\left(\tau_{C_{6}}=+\infty\right)=0 \text { for } \quad|x|>C_{6} .
$$

Thus

$$
P_{(x, \mathbf{a})}\left(\tau_{C_{6}}<+\infty\right)=1 .
$$

On the other hand, it is easy to show

$$
\inf _{\substack{|x| \leq C_{6} \\ \mathbf{a} \in \mathbb{Z}^{2}}} P_{(x, \mathbf{a})}\left(X_{t}=0 \text { for some } t>0\right)>0 .
$$

This combined with an argument in the proof of Lemma 1.1, [2] shows

$$
P_{(x, \mathbf{a})}\left(X_{t}=0 \quad \text { for some } \quad t>0\right)=1 .
$$

Thus the proof of Theorem 4 is complete.

Acknowledgements. The author would like to thank the referee for some suggestions.

\section{REFERENCES}

[1] S. N. Ethier and T. G. Kurtz, Markov Processes - Characterization and Convergence, John-Wiley \& Sons, New York, 1986.

[2] K. Ichihara, Some global properties of symmetric diffusion processes, Publ. RIMS, Kyoto Univ., 14 (1978), 441-486.

[3] T. M. Liggett, Interacting particle systems, Springer-Verlag, New York, 1985.

[4] N. Madras, A process in a randomly fluctuating environment, Ann. Prob., 14 (1986), 119-135.

Department of Mathematics

Faculty of Engineering

Kansai University

Suita-shi, Osaka 565-8680

Japan

kchihara@ipcku.kansai-u.ac.jp 